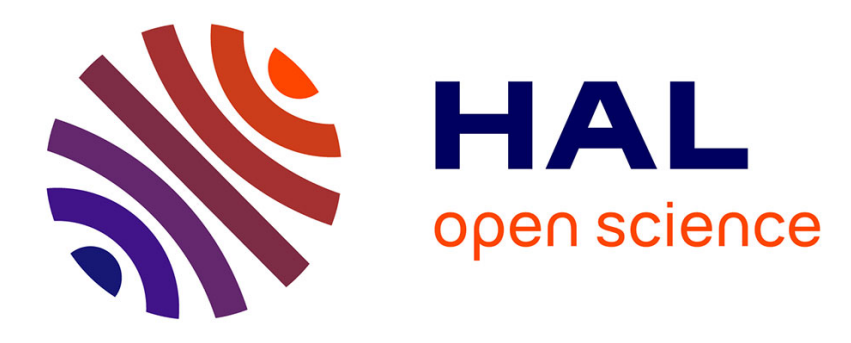

\title{
La question de la concordance des temps en russe
}

Christine Bracquenier

\section{To cite this version:}

Christine Bracquenier. La question de la concordance des temps en russe. Langages, 2013, La concordance des temps, 191, pp.81-94. halshs-00731800

\section{HAL Id: halshs-00731800 \\ https://shs.hal.science/halshs-00731800}

Submitted on 13 Sep 2012

HAL is a multi-disciplinary open access archive for the deposit and dissemination of scientific research documents, whether they are published or not. The documents may come from teaching and research institutions in France or abroad, or from public or private research centers.
L'archive ouverte pluridisciplinaire $\mathbf{H A L}$, est destinée au dépôt et à la diffusion de documents scientifiques de niveau recherche, publiés ou non, émanant des établissements d'enseignement et de recherche français ou étrangers, des laboratoires publics ou privés. 
Christine Bracquenier

MoDyCo, UMR 7114

Université Charles-de-Gaulle - Lille 3

CNRS - Université Paris Ouest Nanterre La Défense

\section{Version de travail}

\section{La question de la concordance des temps en russe}

Résumé : Christine Bracquenier, «La question de la concordance des temps en russe » Le russe ne connaît pas la « concordance des temps » qui oblige à modifier le temps du verbe subordonné en fonction de celui du verbe régissant. Le présent dans une subordonnée du discours indirect ou dans une complétive autre situe le procès en simultanéité par rapport au moment régissant quel que soit son temps, le futur exprime un procès postérieur et le prétérit, un procès antérieur. Or, l'emploi des formes aspecto-temporelles dans les phrases complexes n'est pas si simple. Le choix de l'aspect joue un rôle non négligeable, s'avérant discriminant pour le passé. Les complétives introduites par čto [que] doivent être distinguées des relatives sans antécédent dont le temps «s'accorde » avec celui du verbe régissant; les complétives des verbes de perception fonctionnent comme des relatives; celles des verbes de volition sont à un mode virtuel. Dans les phrases conditionnelles, les deux verbes sont soit à la même forme, soit chacun d'eux en accord avec le temps du monde réel. Ainsi, si le temps du verbe régissant n'a pas d'influence sur celui du verbe subordonné, d'une part sa sémantique, d'autre part la nature des relations syntaxiques entre la régissante et la subordonnée sélectionnent la forme du verbe subordonné et je dirais qu'en russe il s'agit plutôt d'accordance que de concordance.

Mots-clés : russe - système aspecto-temporel - propositions complétives, relatives, conditionnelles - discours indirect - accordance.

Abstract : Christine Bracquenier, «The question of tense agreement in Russian»

Tense agreement does not exist in Russian as it does in French: the verb tense in the main clause does not influence the tense in the subordinate clause. But this question is not so simple and there are some cases where the verbal forms seem to be in competition. In fact, the analysis of verb Aspect shows the differences. The noun clauses introduced by čto [that] have to be distinguished from the relative clauses in which the verb form is in accordance with the verb of the main clause. It is the same when the main verb is a verb of perception. When the main verb is a volition verb, the subordinate verb is in the virtual mood. In the conditional phrases either verbs are in the same form or their tenses are chosen in agreement with the real time. So, the tense of the main verb has no influence on the tense of the subordinate verb in Russian, but the semantics of the verb and the nature of the syntactic relations between main and subordinate clauses select the form of the subordinate verb. It is rather "accordance" than "concordance".

Key words: Russian - tense and aspect - noun, relative, conditional clauses - indirect speech - "accordance".

Si l'on entend par «concordance des temps » le phénomène grammatical qui oblige à modifier le temps-tiroir du verbe subordonné en fonction de celui du verbe régissant ${ }^{1}$, qui contraint le français à dire :

\footnotetext{
${ }^{1}$ J'utilise les termes «verbe régissant» et «proposition régissante » à la place de «verbe principal » et «proposition principale» dans la mesure où le verbe qui m'intéresse peut dépendre d'une proposition déjà
} 
(a) Il dit qu'elle arrive $v s$ Il a dit / il dit / il disait qu'elle arrivait ${ }^{2}$;

(b) Il dit / il pense qu'elle viendra $v s$ Il a dit / il pensait qu'elle viendrait ;

(c) Il ne sait pas si elle viendra $v s$ Il ne savait pas si elle viendrait ;

(d) Il dit qu'elle est partie $v s$ Il a dit qu'elle était partie ;

dans ce cas, effectivement, le russe ne connaît pas la «concordance des temps », ce que montre la traduction de ces exemples :

(a') Он говорит, что она приходит vs Он сказал / говорил, что она приходит;

On govorit, čto ona prixodit vs On skazal/govoril, čto ona prixodit.

Il dit que elle arrive $v s$ Il a-dit / disait que elle arrive.

(b') Он говорит / думает, что она придет vs Он сказал / думал, что она придет;

On govorit / dumaet, čto ona pridet vs On skazal/dumal, čto ona pridet.

Il dit / pense que elle viendra $v s$ Il a-dit / pensait que elle viendra.

(c') Он не знает, придет ли она vs Он не знал, придет ли она;

On ne znaet, pridet li ona vs On ne znal, pridet li ona.

Il ne sait viendra est-ce-que elle $v s$ Il ne savait viendra est-ce-que elle.

(d') Он говорит, что она ушла vs Он сказал / говорил, что она ушла.

On govorit, čto ona ušla vs On skazal/govoril, čto ona ušla.

Il dit que elle est-partie vs Il a-dit / disait que elle est-partie.

[analyse des exemples ci-dessus]

Afin de simplifier la tâche des apprenants francophones, il est usuel de leur dire qu'il suffit d'employer en russe dans les subordonnées complétives et interrogatives indirectes le temps-tiroir du style direct, et les traditionnels, mais peu réalistes, exercices structuraux qui consistent à transformer le discours direct en discours indirect ne présentent qu'un intérêt tout à fait relatif, même si l'on fait varier le temps du verbe de la proposition régissante.

La situation semble être si claire que ce phénomène de la «concordance des temps » n'est pas même évoqué dans la majorité des grammaires françaises du russe (Mazon, Garde, Comtet, Kor Chahine \& Roudet, etc.) ni dans les grammaires russes (Valgina, Dibrova, Grammaires de l'Académie, etc. $)^{3}$; la question n'est guère abordée que dans des ouvrages de grammaire comparative (Gak, Kuznecova, Boulanger).

Ainsi, en russe, dans une phrase complexe dont la subordonnée est une complétive ou une interrogative indirecte, c'est le moment régissant qui permet de savoir où se place l'ensemble $<$ régissant + régi $>$ sur l'axe du temps, le temps-tiroir de la subordonnée indiquant seulement si cet acte est antérieur, simultané ou postérieur à l'acte régissant. En ce sens, il n'y a pas de concordance des temps en russe. Cela peut s'expliquer par la morphologie verbale du russe qui ne possède que cinq formes temporelles finies réparties sur les deux aspects : présent imperfectif, présent perfectif (cf. note 3), futur imperfectif, prétérit imperfectif, prétérit perfectif. Le choix des temps-tiroirs est restreint, les possibilités de « concordance » le sont de facto. En revanche, le russe use de son système aspectuel, dont la primauté sur le système temporel est à prendre en considération, et nous verrons que le choix de l'aspect joue un rôle important dans les relations interpropositionnelles.

Les choses ne sont donc pas si simples.

subordonnée, et aussi parce que souvent la «proposition principale» ne contient pas l'information «principale ».

2 À l'instar du «conditionnel présent » (cf. ex. (b)) appelé de nos jours « futur du passé » auquel Kuznecova consacre un paragraphe (p. 125-136), elle nomme, en toute logique, l'imparfait français de la subordonnée de (a) «le présent du passé » (p. 112). Il semble cependant que sa proposition terminologique n'ait pas retenu l'attention des grammairiens. (Voir ici même la remarque de Romana Bardy à ce sujet).

${ }^{3}$ On comprend aisément que la «concordance des temps » ne soit pas abordée en tant que telle dans les grammaires russes du russe puisque ce phénomène n'est pas considéré comme pertinent pour la syntaxe russe. 


\section{1. «Concurrence » présent vs passé pour l'expression de la simultanéité \\ 1.1. Simultanéité vs antériorité}

[...] Si l'expression de la simultanéité n'est pas totalement exclue, elle n'est cependant jamais portée par la forme verbale, mais doit être précisée par le cotexte et le contexte, la valeur par défaut étant celle de l'antériorité d'un procès isolé dans le passé. Je ne peux accepter l'analyse de Xomicevič (2006 : 466) qui cite (1) afin de démontrer que le prétérit subordonné indique aussi (comme le présent) une simultanéité par rapport à un acte régissant passé :

$$
\begin{array}{llll}
\text { Mы } & \text { знали [Iра], } & \text { что Мария жила [Iра] в } & \text { общежитии } \\
\text { My znali [Ipa], } & \text { čto Marija žila [Ipa] } & \text { v } & \text { obŝežitii. } \\
\text { Nous savions } & \text { que Maria vivait } & \text { dans } & \text { résidence-universitaire. }
\end{array}
$$

Nous savions que Maria vivait / avait vécu à la/dans une/en résidence universitaire.

En dehors de tout contexte, cet exemple est certes interprétable [...] On comparera (1) à (2) où le prétérit imperfectif exprime un procès antérieur au procès régissant et sans lien temporel avec lui et (3) où le présent dénote un procès simultané au procès régissant ${ }^{5}$ :

(2) В разговоре я вспомнил [Рра], что уж один раз и Матрена сама почему-то ходатайствовала [Іра] за Антошку Григорьева, но я не спросил, что за родственник он ей, и тоже тогда отказал. (А. Солженицын, Матренин двор)

$\mathrm{V}$ razgovore â vspomnil [Ppa], čto už odin raz i Matrena sama počemu-to xodatajstvovala [Ipa] za Antošku Grigor'eva, no â ne sprosil, čto za rodstvennik on ej, i tože togda otkazal. (Solženicyn)

Pendant la conversation, je me souvins qu'une fois déjà Matriona elle-même était intervenue en faveur du petit Anton Grigoriev, mais je n'avais pas demandé quel était leur lien de parenté et déjà alors j'avais refusé. 6

(3) Ему казалось [Iра], что через землю он слышит [Ipr] запах свежего хлеба. (А. Приставкин, Ночевала тучка золотая)

Emu kazalos' [Ipa], čto čerez zemlû on slyšit [Ipr] zapax svežego xleba. (Pristavkin)

Il lui semblait qu'il sentait l'odeur du pain frais à travers la terre.

\subsection{Complétive vs relative}

L'examen attentif du corpus montre que lorsque la subordonnée introduite par čto [que] comporte un prétérit imperfectif qui n'a de toute évidence pas de valeur antérieure, il s'agit en réalité non pas d'une complétive, mais d'une relative sans antécédent où čto est alors pronom relatif sujet ou objet du verbe de la subordonnée. On peut ainsi différencier :

(4a) Он сказал [Рра], что думает [Ipr]

On skazal [Ppa], čto dumaet [Ipr]

Il a-dit que pense

Il a dit qu'il pensait/réfléchissait.

et

(4b) Он сказал [Рра], что думал [Iра]

On skazal [Ppa], čto dumal [Ipa]

Il a-dit que pensait

Il a dit ce qu'il pensait.

\footnotetext{
${ }^{4}$ Désormais : $[\mathrm{pr}]=$ présent $;[\mathrm{Ipr}]=$ imperfectif présent $;[\mathrm{Ppr}]=$ perfectif présent (à valeur de futur) ; [Ifut $]=$ imperfectif futur ; [Ipa] = imperfectif prétérit ; [Ppa] = perfectif prétérit ; [Iinf] = imperfectif infinitif ; [Pinf $]=$ perfectif infinitif] ; [Icond] = imperfectif conditionnel ; [Pcond] = perfectif conditionnel.

${ }^{5}$ L'aspect du verbe régissant n'a ici pas d'influence sur la forme du verbe régi. Le prétérit perfectif subordonné dénotant toujours un procès antérieur au moment régissant, je ne donne pas d'exemple.

6 Les exemples, accompagnés d'un nom d'auteur sont tirés du Corpus national de la langue russe (ruscorpora.ru). Le corpus que j'étudie est la langue littéraire contemporaine. La langue de la presse fonctionne de la même manière. Toutes les traductions sont faites par mes soins.
} 
En effet, le verbe d'une proposition relative qui exprime un procès simultané à celui de la proposition régissante au prétérit se conforme au temps de la régissante et se met également au prétérit, «quel que soit le mot qui [l'] introduit $7^{7}$. Je parlerais alors d'accordance des temps ${ }^{8}$. Ainsi, en (4a) le présent du verbe subordonné oblige à une interprétation conjonctive de čto, alors que le prétérit de $(4 \mathrm{~b})$ indique que l'on a affaire au pronom relatif sans antécédent. Des exemples tirés du corpus confirment cette observation ${ }^{9}$ :

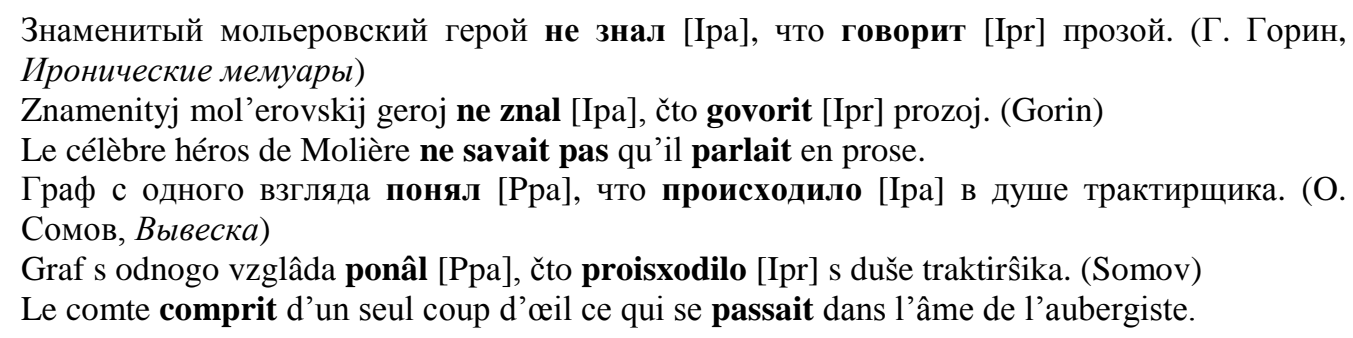

En (5) l'interprétation de čto comme relatif est exclue par le présent de la subordonnée, ce qui est corroboré par le sens de la phrase (?Le héros de Molière ne savait pas ce qu'il disait en prose). En (6), au contraire, le prétérit d'une part, l'absence de sujet autre que čto d'autre part ne permet pas de considérer čto autrement que comme relatif. Cependant quelques exemples semblent contredire ce que je viens d'affirmer concernant ces relatives sans antécédent ; en effet, ils comportent un verbe au présent, comme en (7) :

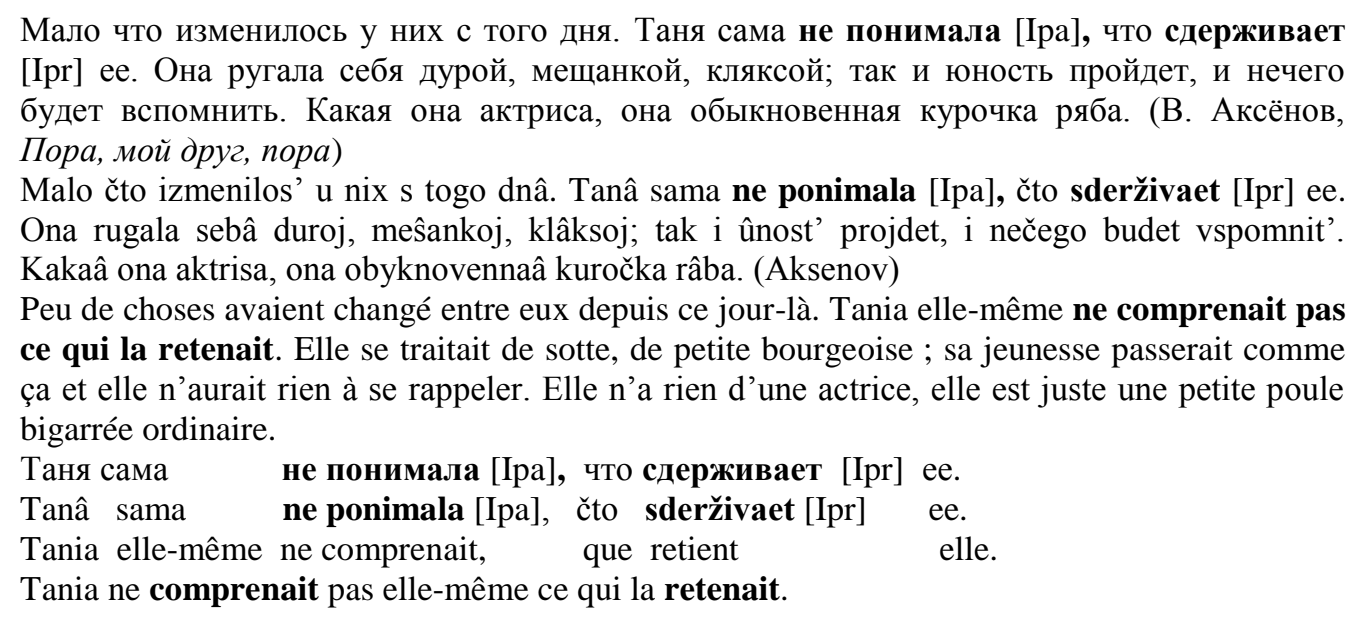

Cet exemple, si l'on n'y prend garde, peut être interprété comme <régissante + complétive ${ }^{10}$ et l'on y trouve les formes aspecto-temporelles d'une phrase complexe dont les deux procès sont simultanés. Cependant, la deuxième proposition est bien une proposition relative. Alors pourquoi l'attraction temporelle n'a-t-elle pas joué ? Il faut regarder le cotexte droit pour comprendre que cette proposition fait passer le texte de la narration au discours indirect libre et c'est le temps de la relative qui opère ce décrochement dans le schéma du texte : on passe ainsi de la voix du narrateur (qui utilise le passé de narration) à celle du personnage (qui utilise un présent d'énonciation).

\footnotetext{
${ }^{7}$ M. Guiraud-Weber $1974: 70$.

${ }^{8} \mathrm{Si}$ mes lecteurs me permettent ce néologisme sémantique.

${ }^{9}$ Aucun ouvrage grammatical ni aucune recherche, à ma connaissance, ne mettent en évidence ce fait.

${ }^{10}$ En effet, M. Guiraud-Weber (1974: 74) qui donne l'exemple que je cite en (7), considère la subordonnée comme une complétive.
} 


\subsection{Les complétives des verbes de perception}

Il convient également de s'intéresser à l'emploi des formes aspecto-temporelles des subordonnées régies par un verbe de perception. [...] :

(8a) Я видела [Iра], как дети играют [Ipr]/играли [Iра] в мяч.

Â videla [Ipa], kak deti igraût [Ipr] /igrali [Ipa] v mâč.

Je ai-vu comment enfants jouent /jouaient dans ballon.

J'ai vu des/les enfants jouer au ballon.

(8b) Я видела [Iра], что дети играют [Ipr]/играли [Ipa] в мяч.

Â videla [Ipa], čto deti igraût [Ipr] /igrali [Ipa] v mâč.

Je ai-vu que enfants jouent /jouaient dans ballon.

J'ai vu que des/les enfants jouaient au ballon.

(8c) Я видела [Ipa] детей, играющих [part. pr]/игравших [part. pa] в мяч.

Â videla [Ipa] detej, igraûŝix [part. pr] /igravšix [part. pa] v mâč.

Je ai-vu enfants jouant djouant dans ballon.

J'ai vu des/les enfants qui jouaient au ballon.

(8d) Я видела [Iра] детей, которые играли [Iра] в мяч.

Â videla [Ipa] detej, kotorye igrali [Ipa] $\mathrm{v}$ mâč.

Je ai-vu enfants qui jouaient dans ballon.

J'ai vu des/les enfants qui jouaient au ballon. ${ }^{11}$

En (8), quelle que soit la structure, à l'exception toutefois de (8d), on constate une soidisant concurrence entre le présent et le prétérit imperfectif. (8d) comportant une relative, la seule forme possible est le prétérit, ce qui peut être confirmé par les exemples attestés suivants, où (9) est au présent parce qu'il s'agit d'une complétive et où (10) ne peut être qu'au prétérit dans la relative (voir aussi ci-dessus) :
Он видел [Іра], что не нравится [Ipr] тёще, и это его сковывало. (В. Токарева, Своя правда)
On videl [Ipa], čto ne nravitsâ [Ipr] teŝe, i èto ego skovyvalo. (Tokareva)
Il voyait qu'il ne plaisait pas à sa belle-mère et cela le paralysait.
I â videl, čto tvorilos's nim. (Kio)
Et j'ai vu ce qui lui arrivait.
И я видел [Іра], что творилось [Іра] с ним. (И. Кио, Иллюзии без иллюзий)

Cependant, le prétérit et le présent peuvent être utilisés tous deux dans les complétives. Jean Breuillard (2008) étudie les emplois des participes présent actif et passé actif imperfectifs dans des contextes passés. Ces deux formes sont en effet, elles aussi, souvent caractérisées comme concurrentielles, or l'auteur montre qu'il n'en est rien :

Tout se passe donc comme si, en système de récit, le narrateur pouvait changer le point d'observation librement. L'action peut être décrite de l'extérieur, par le narrateur. L'action est alors prise dans le flux narratif, dans la séquence narrative à laquelle Jean-Paul Sémon a donné le nom de chronopoï̀se. Elle y occupe une place. Le Pa [participe passé] est la marque de cette insertion.

Mais l'action peut être présentée comme vue par le personnage. Le Pr [participe présent] est alors employé. Ce que voit le personnage est une action toujours présente et concomitante à son propre temps. Ce que décrit le narrateur est au contraire une action qu'il situe par rapport au temps du récit ${ }^{12}$.

J. Breuillard conclut sur la marque de l'empathie exprimée par le présent. Ses observations se vérifient dans le cas des complétives des verbes de perception ; en (11) et (12) le présent oblige l'énonciataire à découvrir la situation dans le présent du personnage et en (12) cette empathie est même verbalisée :

\footnotetext{
${ }^{11}$ Je ne pose pas ces énoncés comme "synonymes »; ils présentent la situation décrite de manière différente, mais ce n'est pas mon propos ici. La différence entre les conjonctions čto et kak nécessite une étude particulière.

${ }^{12}$ J. Breuillard $2008: 252$.
} 
Дед Иван жил [Іра] так уже давно и, всё больше теряя зрение, не видел [Іра], что его квартира становится [Ipr] местом военных действий - армия пауков против многочисленных армий мух. (Л. Петрушевская, Маленькая волиебница)

Ded Ivan žil [Ipa] tak uže davno i, vse bol'še terââ zrenie, ne videl [Ipa], čto ego kvartira stanovitsâ [Ipr] mestom voennyx dejstvij - armiâ paukov protiv mnogočislennyx armij mux. (Petruševskaâ)

Le vieil Ivan vivait ainsi depuis longtemps et, perdant de plus en plus la vue, il ne voyait pas que son appartement devenait un champ de guerre: une armée d'araignées contre d'innombrables armées de mouches.

(12) Колюнчику было очень жалко папу, он видел [Iра], как тот старается [Ipr] и страдает [Ipr], [...]. (А. Варламов, Купавна)

Kolûnčiku bylo očen' žalko papu, on videl [Ipa], kak tot staraetsâ [Ipr] i stradaet [Ipr], [...]. (Varlamov)

Le petit Colas avait grande pitié de son papa, il voyait qu'il faisait tout son possible et souffrait, $[\ldots]$.

En revanche, $[\ldots]$

(13) В бинокль я видел [Іра], как он выходил [Іра] из чащи. (Д. Гранин, Зубр)

V binokl' â videl [Ipa], kak on vyxodil iz čaŝi. (Granin)

Avec les jumelles, je le voyais sortir du fourré (litt. Je voyais qu'il sortait).

(14) Когда я поднялся по лестнице ...ского облоно и спросил, где отдел кадров, то с удивлением увидел [Рра], что кадры уже не сидели [Іра] здесь за черной кожаной дверью, а за остекленной перегородкой, как в аптеке. (А. Солженицын, Матренин двор) Kogda â podnâlsâ po lestnice ...skogo oblono i sprosil, gde otdel kadrov, to s udivleniem uvidel [Ppa], čto kadry uže ne sideli [Ipa] zdes' za černoj kožanoj dver'û, a za osteklennoj peregorodkoj, kak v apteke. (Solženicyn)

Quand j'eus monté l'escalier de l'Office régional de l'Instruction publique de la région de N. et demandé où se trouvait le service du personnel, je m'aperçus avec étonnement que les fonctionnaires n'étaient plus installés derrière une porte capitonnée de cuir noir, mais derrière une cloison en verre, comme dans les pharmacies.

(15) Вообще, приглядываясь к Матрене, я замечал [Іра], что, помимо стряпни и хозяйства, на каждый день у нее приходилось [Іра] и какое-нибудь другое немалое дело, [...] (А. Солженицын, Матренин двор)

Voobŝe, priglâdyvaâs' k Matrene, â zamečal [Ipa], čto, pomimo strâpni i xozâjstva, na každyj den' u nee prixodilos' [Ipa] i kakoe-nibud' drugoe nemaloe delo, [...]. (Solženicyn)

En fait, en observant Matriona, je remarquais qu'outre la cuisine et les travaux domestiques, elle avait quelque autre tâche, tout aussi importante, prévue pour chaque jour.

Le prétérit peut aussi s'expliquer, dans cette configuration, par son caractère anaphorique, ce qui n'est pas le cas du présent, et le temps employé est très certainement lié à la définitude des syntagmes nominaux de la complétive, mais ce n'est pas là l'objet de la présente recherche.

\section{Les complétives des verbes de volition}

Les complétives des verbes de volition comportent en français un subjonctif. Ce mode n'est pas reconnu comme existant en russe et les grammaires se contentent de dire qu'après un verbe de volition il faut remplacer la conjonction čto par čtoby ${ }^{13}$ et mettre le verbe au prétérit. Un verbe au prétérit accompagné de la particule verbale by correspond à ce que les

\footnotetext{
${ }^{13}$ Il s'agit de la particule verbale by qui s'est, au fil du temps, agglutinée à la conjonction. Cette particule provient d'une ancienne forme de $2^{\mathrm{e}}$ et $3^{\mathrm{e}}$ personnes du singulier de l'aoriste du verbe byt' [être] employée comme auxiliaire de l'aoriste parfait du vieux russe qui exprimait « une action passée comme abolie dans son résultat. D'où un glissement précoce vers la signification hypothétique » (Veyrenc $1970: 7$ ).
} 
grammaires du russe appellent le conditionnel ${ }^{14}$. Il serait, selon moi, beaucoup plus raisonnable d'appeler ce mode le « virtuel ». Cela éviterait le flou d'un subjonctif qui n'a pas de réalité formelle et il ne serait pas très économique de poser un subjonctif homonyme du conditionnel puisque le «subjonctif» n'apparaît qu'avec la conjonction čtoby dans une subordonnée complétive d'un verbe de volition ${ }^{15}$. Pour ce qui est de la concordance des temps avec une régissante de volition, la question ne se pose donc pas. Ne subsiste que l'aspect, et le corpus montre que le choix de l'aspect du verbe subordonné n'est lié ni au temps ni à l'aspect du verbe régissant.

\section{Les phrases conditionnelles}

Dans les phrases conditionnelles ${ }^{16}$, en russe, la protase et l'apodose présentent souvent le même temps-tiroir ou le même mode: deux futurs pour une relation de cause à effet potentielle ${ }^{17}$, cf. (16) ; deux présents pour une relation cause-effet éventuelle, unique ou itérative et donc atemporelle; deux prétérits pour une relation de cause à effet réalisée dans le passé ; deux conditionnels pour une relation dans l'irréel du présent ou du passé (17). Dans tous ces cas, je ne pense pas qu'on puisse parler d'une concordance des temps ; en effet, les deux situations se placent dans le même espace temporel réel ou irréel (présent, passé ou futur) dénoté par le temps-tiroir. Il y a « accordance » des temps, mais aucun des deux verbes n'impose sa forme à l'autre.

(16) Я его не впущу [Ppr], если он придет [Ppr]... (И. Эренбург, Оттепель)

Â ego ne vpuŝu [Ppr], esli on pridet [Ppr]... (Ërenburg)

Je ne le laisserai pas entrer s'il vient...

Если бы он пришел [Pcond] к вам и сказал ${ }^{18}$ [Pcond]: «Тоня, прости меня», — вы бы не пожалели [Pcond]? (В. Кетлинская, Мужество)

Esli by on prišel [Pcond] k vam i skazal [Pcond]: «Tonâ, prosti menâ», - vy by ne požaleli [Pcond]? (Ketlinskaâ)

S'il était venu vous voir et vous avait dit : «Tonia, pardonne-moi », vous n'auriez pas eu pitié ?

Mais cette unité modo-temporelle n'est pas absolue et dans de nombreux cas les deux propositions ne comportent pas la même forme verbale. Il ne s'agit pas non plus, cependant, d'une concordance des temps « à la française ». Il faut ici distinguer deux cas de figure :

- le cas où les formes verbales sont différentes, mais situent les procès dans le même espace modo-temporel, par exemple quand la protase comporte un «impératif syntaxique ${ }^{19}$ (18) ou un infinitif ou quand le verbe de l'apodose, dans une relation potentielle, est à l'impératif :

\footnotetext{
${ }^{14}$ Il n'existe qu'un temps au conditionnel, mais le conditionnel peut se former sur les deux aspects (verbe au prétérit $+b y)$. La particule peut également s'ajouter à l'infinitif de chacun des deux aspects pour former un conditionnel dit impersonnel.

${ }^{15} \mathrm{Ou}$ équivalent sémantique. La proposition régissante peut aussi être en ellipse dans l'expression d'un souhait ou d'un ordre : Čtoby on prišel ! [Qu'il vienne !].

${ }^{16}$ Le propos n'est pas de mener ici une étude des phrases conditionnelles, mais de présenter leur fonctionnement en termes de système aspecto-modo-temporel.

${ }^{17}$ Pour la terminologie voir C. Touratier 2008. Le russe ne possède plus de temps verbaux composés comme le futur antérieur ou le plus-que-parfait ; ainsi dans les phrases «Si tu termines /as terminé avant huit heures, nous irons au restaurant » et « Si tu réussis / as réussi, tu auras remporté une belle victoire » (A. Bentolila 1995 : 289), toutes les formes verbales du français seront traduites en russe par un futur.

${ }^{18}$ La particule by est en ellipse dans la coordonnée.

${ }^{19} \mathrm{Il}$ s'agit de la $2^{\mathrm{e}}$ personne du singulier de l'impératif, devenue invariable et ayant perdu la notion de personne ; cette forme dessert les valeurs d'irréel du présent, du passé et du futur. Elle est également appelée «quasiimpératif » (par les grammaires russes) ou encore « conditionnel syntaxique ».
} 
Pridi [Pimp] on v kliniku vmesto menâ, i ty segodnâ byla by [Icond] sčastliva. (Zorin)

S'il était venu à l'hôpital à ma place, toi aussi tu serais heureuse aujourd'hui.

Il ne faut pas se laisser abuser par des effets de surface, où l'on peut avoir l'impression d'une concordance des temps semblable à celle du français (cf. (19) : prétérit dans la protase otorvalsâ, et conditionnel dans l'apodose - žil by), mais il ne s'agit que d'une ellipse de la particule by. Cela s'observe lorsque le cotexte gauche est déjà noté explicitement comme relevant de l'irréel ${ }^{20}$. Ce phénomène, relativement peu fréquent en subordination, est néanmoins intéressant et est peut-être l'indice d'un changement systémique de la langue :
Ирина отчитала Павла. Он сорвал всю схему. Девочка бы росла, Володька бы привыкал и, возможно, оторвался от армянки. А если не оторвался [Pcond sans by exprimé], жил бы [Icond] на два дома. Всё лучше, чем ничего. (В. Токарева, Своя правда)
Irina otčitala Pavla. On sorval vsû sxemu. Devočka by rosla, Volod'ka by privykal i, vozmožno, otorvalsâ ot armânki. A esli ne otorvalsâ [Pcond sans by exprimé], žil by [Icond] na dva doma. Vse lučše, čem ničego. (Tokareva)
Irina fit la leçon à Pavel. Il avait détruit tout leur plan de vie. La fillette aurait grandi, Volodia se serait habitué et sans doute séparé de son Arménienne. Et s'il ne s'en était pas séparé, il aurait vécu dans deux maisons. Ça aurait été mieux que rien.

- le cas où les formes verbales sont différentes parce qu'elles correspondent à un espace temporel différent ; en (20) le russe emploie le présent d'énonciation pour le verbe régissant suivi d'un présent perfectif limitatif ${ }^{21}$ à valeur de futur, dans la mesure où le bourdon n'a pas encore commencé son travail : l'acte de travailler est postérieur à la fois à l'énonciation et aux récriminations possibles des abeilles :

(20) - Шм-шм-шм,- пробасил Шмель, обращаясь к пчёлкам. - Вы не возражаете [Ipr], если я тут поработаю [Ppr]? Но пчёлки почему-то Шмеля не услышали. (В. Кологрив, Медовый луг)

- Šm-šm-šm, - probasil Šmel', obraŝaâs' k pčelkam. - Vy ne vozražaete [Ipr], esli â tut porabotaû $[\mathrm{Ppr}]$ ? No pčelki počemu-to Šmelâ ne uslyšali. (Kologriv)

- Bzz-bzz-bzz, dit Bourdon de sa voix de basse, s'adressant aux petites abeilles. - Cela ne vous dérange pas si je travaille un peu ici ? (litt. Vous n'objectez pas si je *travaillerai-un-peu ici). Mais, on ne sait pourquoi, les petites abeilles n'entendirent pas Bourdon.

En (21) et (22) la condition est réalisée et le verbe subordonné est un prétérit perfectif, à valeur parfaite en (21) et le présent de la régissante s'insère, en quelque sorte, dans le flux temporel de la condition ; en (22) il marque l'antériorité, et la conséquence exprimée par un « futur » perfectif (sdelaet) est postérieure à la fois à ce procès et à l'énonciation :

(21) Если Америку из кризиса вывел [Рра] президент-актёр, нам необходим [pr] режиссёр. (Г. Горин, Иронические мемуары)

Esli Ameriku iz krisica vyvel [Ppa] president-akter, nam neobxodim [pr] - režisser. (Gorin)

Si c'est un président-acteur qui a sorti l'Amérique de la crise, nous avons besoin d'un metteur en scène.

(22) Лизавета наверняка знала, что Игорь Горный слов на ветер не бросает и если сказал [Рpa], что выяснит [Ppr], то сделает [Ppr] непременно. (Е. Козырева, Дамская охота)

Lizaveta navernâka znala, čto Igor' Gornyj slov na veter ne brosaet i esli skazal [Ppa], čto vyâsnit [Ppr], to sdelaet [Ppr] nepremenno. (Kozyreva)

Elisabeth savait de manière certaine que Igor Gorny ne lançait pas des paroles en l'air et que s'il avait dit qu'il tirerait les choses au clair, il le ferait sans faute (litt. *s'il avait dit qu'il tirera les choses au clair, il le fera).

\footnotetext{
${ }^{20}$ Cf. (17).

${ }^{21}$ C'est le préverbe po- qui porte la valeur limitative.
} 
En (23) les menaces relèvent du plan de la narration menée au passée (ugrožal), le procès envisagé (la mise en œuvre des menaces), exprimé par un infinitif perfectif (perestrelât'), est postérieur au «dire » des menaces; celles-ci ne seront exécutées que dans le cas, lui aussi postérieur aux menaces, où les hommes «se défileraient » (sdrejfât-perfectif présent à valeur de futur). Il y a donc trois «moments» successifs : les menaces, le manque de courage éventuel, la fusillade éventuelle. La postériorité au moment régissant quel qu'il soit est toujours exprimée par un futur :

(23) Трактирщик тем временем не умолкал - то командовал сорванным голосом, то увещевал почти ласково. А то угрожал [Ipa] перестрелять [Pinf] всех, если сдрейфят [Ppr]. (В. Быков, Камень)

Traktirŝik tem vremenem ne umolkal - to komandoval sorvannym golosom, to uveŝeval počti laskovo. A to ugrožal perestrelât' vsex, esli sdrejfât. (Bykov)

L'aubergiste cependant ne cessait de parler, tantôt il commandait de sa voix cassée, tantôt il les exhortait presque amicalement. Ou encore il menaçait de les descendre tous s'ils se défilaient.

Lorsque les plans temporels de la régissante et de la subordonnée sont différents, les temps-tiroirs situent chacun des procès sur l'axe du temps en prenant pour repère le moment du dire, que celui-ci se trouve dans le présent (20) ou le passé (23). Il n'y a donc pas de concordance des temps.

\section{Conclusion}

Lorsqu'il s'agit d'étudier les rapports entre les formes aspecto-temporelles de phrases complexes, il est nécessaire de prendre en considération de nombreux critères, parmi lesquels, en premier lieu, l'ordonnancement chronologique des procès en jeu, mais aussi la sémantique du verbe régissant, la nature réelle de la subordonnée. Les exemples cités ici montrent aussi combien il est important de tenir compte de la situation de production de l'énoncé : s'il s'agit d'une narration détachée du moment d'énonciation, d'un discours au sens de Benveniste, ou encore d'un discours indirect libre. Les cotextes gauche et droit influent également de manière notable sur le choix du temps-tiroir de la subordonnée. L'analyse de ces différents éléments permet d'approfondir la question de l'emploi des temps-tiroirs dans les phrases complexes, et de nuancer l'affirmation selon laquelle il n'y a pas de concordance des temps en russe. Il existe bien une «concordance des temps », mais cette concordance ne peut certainement pas se limiter aux temps. Il s'agit aussi d'une « concordance des modes », et dans certains cas, on peut parler d'une «concordance des aspects». Il serait préférable sans doute d'utiliser le terme d' " accordance des temps », proposé ici, pour décrire ce phénomène en russe.

\section{Bibliographie}

Bentolila A. (dir.) (1995), Grammaire alphabétique, Paris : Le Robert \& Nathan.

BERNITSKAÏA N. (2008), L'ordre temporel en russe contemporain: vers une approche pragmatique du marquage des relations chronologiques, Paris IV : thèse de doctorat inédite.

Boulanger A. (2000), Pratique de la traduction russe, première partie: Éléments de syntaxe comparée, Paris : Ophrys.

BRACQUENIER C. (2008), «Kogda et la construction du temps », in Breuillard \& S. Aslanoff (éds), Construire le temps, Études offertes à Jean-Paul Sémon, Paris : Institut d'Études slaves, 317-336.

BRACQUENIER C. (2010), «Concordance(s) des temps en russe contemporain », in C. Douay (dir.), Système et chronologie, Rennes : PUR, 159-183.

BRACQUENIER C. (2012), «Le présent perfectif en russe : une très grande adaptabilité », in C. Bracquenier \& L. Begioni (dir.), L'aspect dans les langues naturelles: approche comparative, Rennes : PUR, 65-80. 
BREUILLARD J. (2008), « La concurrence 'Participe présent actif/Participe passé imperfectif' en russe », in J. Breuillard \& S. Aslanoff (éds), Construire le temps, Études offertes à Jean-Paul Sémon, Paris : Institut d'Études slaves, 239-259.

COMTET R. $\left(2002^{2}\right)$, Grammaire du russe contemporain, Toulouse : Presses universitaires du Mirail.

Dibrova E.I. et al. (2002), Sovremennyj russkij âzyk. Teorija. Analiz âzykovyx edinic, čast' II, Morfologiâ, sintaksis [Le russe contemporain. Théorie. Analyse des unités de langue. II $^{\mathrm{e}}$ partie: Morphologie, syntaxe], Moscou : Academia.

GAK V.G. $\left(2006^{2}\right)$, Russkij âzyk $v$ sopostavlenii s francuzskim [Le russe en comparaison avec le français], Moscou : URSS.

GARDE P. (1998²), Grammaire russe, Phonologie et morphologie, Paris : Institut d'Études slaves.

Grammatika russkogo âzyka (1960), [Grammaire de la langue russe], Moscou : Académie des sciences.

GuIRAUD-Weber M., BARLESI F. (1974), «Y a-t-il une concordance des temps en russe », Les langues modernes, $\mathrm{N}^{\circ} 1,67-78$.

JAKOBSON R. O. (1957), Shifters, verbal categories and the Russian verb, Havard University.

Kor Chahine I., Roudet R. (2003), Grammaire russe, Les structures de base, Paris : Ellipses.

KuZNECOVA I.N. (1987), Praktičeskij kurs sopostavitel'noj grammatiki russkogo $i$ francuzskogo âzykov [Cours pratique de grammaire comparative russe-français], Moscou : Russkij âzyk.

MASLOV Û.S. (1978), «K osnovaniâm sopostavitel'noj aspektologii » [« Fondements d'une aspectologie contrastive »] in Voprosy sopostavitel'noj aspektologii, Leningrad, 4-44.

MAzon A. (1963), Grammaire de la langue russe, Paris : Institut d'Études slaves.

Nacional'nyj korpus russkogo âzyka [Corpus national de la langue russe], Moscou : Académie des Sciences, ruscorpora.ru

PAducEVA E.V. (1996), Semantičeskie issledovaniâ. Semantika vremeni $i$ vida v russkom âzyke, Semantika narrativa [Études sémantiques. La sémantique du temps et de l'aspect en russe, la sémantique du narratif], Moscou, Škola « Âzyki russkoj kul’tury ».

Russkaja grammatika, 1980 [Grammaire russe], Moscou, Académie des sciences.

SEMON J.-P. (1990), «À propos de l'informativité indirecte : l'argumentation et l'aspect en russe », Revue des études slaves, t. 62, fasc. 4., 867-886.

ŠELJAKIN M.A. (2007), Kategoriâ aspektual'nosti russkogo glagola [Catégorie de l'aspectualité du verbe russe], Moscou : URSS.

TOURATIER C. (2008), «Les conditionnelles en français, en russe et dans les langues anciennes », in R. Roudet \& C. Zaremba (éds.), Questions de linguistique slave. Études offertes à M. Guiraud-Weber, Aix-en-Provence, Publications de l'université de Provence, 329-349.

VALGINA N.S. (2000), Sintaksis sovremennogo russkogo âzyka [Syntaxe du russe contemporain], Moscou : Agar.

VEYRENC Ch. J. (1970), Histoire de la langue russe, Paris : P.U.F., Que sais-je ? $\mathrm{N}^{\circ} 1368$.

XoMicEVIC O.G. (2006), «K voprosu o soglasovanii vremen v russkom âzyke » [ Sur la question de la concordance des temps en russe »], $I I^{e}$ colloque international de sciences cognitives, 9-13 juin 2006, Saint-Pétersbourg, cogsci.ru/cogsci06/docs/vol2, 465-466. 